\title{
Medical Research with New-Born Babies: What are the Legal and Ethical Concerns?
}

\author{
Katherine Wade
}

Faculty of Law, UCC

\section{Introduction}

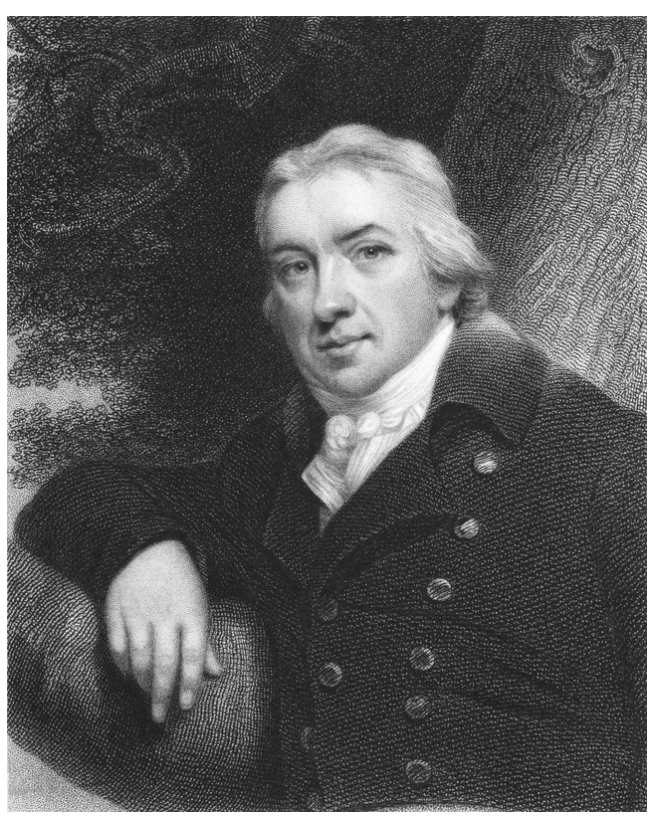

Figure 1: : Edward Jenner

Edward Jenner is accredited with one of the greatest feats of medical science, which is responsible for saving millions of lives. In 1796, he invented the smallpox vaccine. Jenner discovered the vaccine by observing that milkmaids were generally immune to smallpox. He hypothesised that the pus in the blisters that milkmaids received from cowpox protected them from smallpox. He then infected an eight year-old boy with cowpox, which he obtained from the blisters of an infected milkmaid. Afterwards, he injected the boy with smallpox material and the boy showed no sign of infection. He subsequently vaccinated a group of orphan children. The vaccine was also tested on 48 children in an alms-house in Philadelphia. It was through these experiments with children that Jenner created the vaccine for smallpox. While it cannot be denied that these experiments contributed greatly to the progress of medical science and the health of human beings, such a research design would be unlikely to be condoned today. This is because it does not conform to generally accepted ethical standards. Jenner did not conduct any preliminary laboratory research or animal studies. His subjects were particularly vulnerable, being 


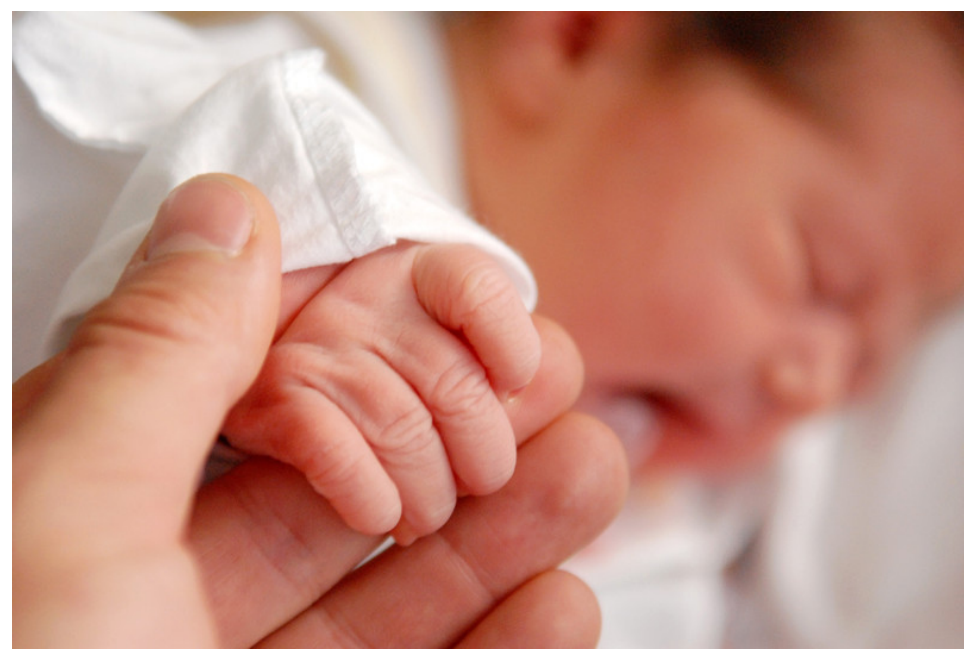

Figure 2: Neonate

under the age of consent. Many of them were institutionalised and orphaned and, thus, lacked parents to make decisions in their best interests. Jenner also deliberately infected healthy children with a disease-causing substance, which was not for their benefit but for that of others. Throughout his experimentation, there was no emphasis on the role of informed consent or the protection of the rights of research subjects.

Medical research ethics has come a long way since 1796. International research ethics were formulated after the atrocities of Nazi experimentation on concentration camp populations came to light. Medical research is now reviewed and regulated by Research Ethics Committees and it must conform to ethical standards laid down in European and international regulation, such as the Nuremberg Code 1947 and the Helsinki Declaration 2008. For example, informed consent is a central requirement in research, there is a right to withdraw at any time, and research must minimise risks for research subjects. Research ethics and regulation seeks to strike a balance between encouraging medical progress and protecting research subjects' rights to life, health, autonomy and dignity.

\section{Why do we need Research with New-Born Babies?}

Medical research with all age-groups, including new-born babies, is important for the progress of medical science. A new-born child or a "neonate" refers to a child in the first 28 days of life. It is crucial that medical research, such as clinical trials, genetic studies and research with medical devices is carried out with this group of children. Neonates are not miniature adults. Their entire physiology differs from adults and so they require drugs and medicines which are suitable for their needs. However, there is a lack of appropriate treatments for neonates. Many medications provide no prescribing information for children. This means that physicians often administer adult treatments to neonates and simply lower the dose of the drug being administered to the infant by guessing its proportionate 
weight relative to an adult. However, as it is impossible to know whether adult-tested drugs will be safe for use with children, this practice could lead to over-dosing, underdosing or specific adverse effects not evident in adults.

Notwithstanding these dangers, such "off-label" or unapproved treatment of neonates occurs on a frequent basis. For example, a European study showed that up to $90 \%$ of neonates in intensive care across Europe receive such prescriptions.

It is often argued that administering unapproved treatments to infants is, in itself, a form of uncontrolled experimentation, as the effects are unknown. It could also be argued that the untested medical care which is given to neonates as a result of "off-label" prescriptions is unethical and a violation of their right to health and life as protected under the Convention on the Rights of the Child 1989. Thus, it is crucial that neonatal medical research is encouraged. However, there are impediments to the encouragement of neonatal medical research. This area poses ethical concerns and thus is seen as difficult to legally regulate. One area which raises particular ethical concerns and which poses particular regulatory challenges is the issue of informed consent.

\section{Informed Consent and Medical Research with New-Borns: What are the Ethical Issues?}

Obtaining informed consent from research participants is a core ethical requirement of medical research. However, as a neonate cannot consent to his or her involvement in research, parents and guardians may give consent on their behalf. There are a number of ethical concerns in this area. The first relates to understanding. In general, research protocols can be difficult to understand, as they can include complicated scientific information and design. However, parental consent in neonatal medical research can pose additional challenges. For example, a mother's ability to make an informed choice may be inhibited, as she could be under the influence of pain relieving medication post-labour. In addition, parents may have just learnt of their neonate's illness, causing stress anxiety and stress. These factors could lead to parents having difficulties understanding the research information and making an uninformed decision about their child's involvement in medical research.

Secondly, parents' understanding can also be affected by therapeutic misconception. This means that they fail to distinguish between treatment and research, and they believe that the research has a wholly therapeutic intent. In particular, this can occur if the researcher who seeks consent is also the treating physician. The parents may assume that the research is in the best interests of the child, since they are being informed about the research from the clinician who is treating their infant. Another worrying aspect of this situation is that parents might feel obliged to consent to research. They may not want to be seen as 


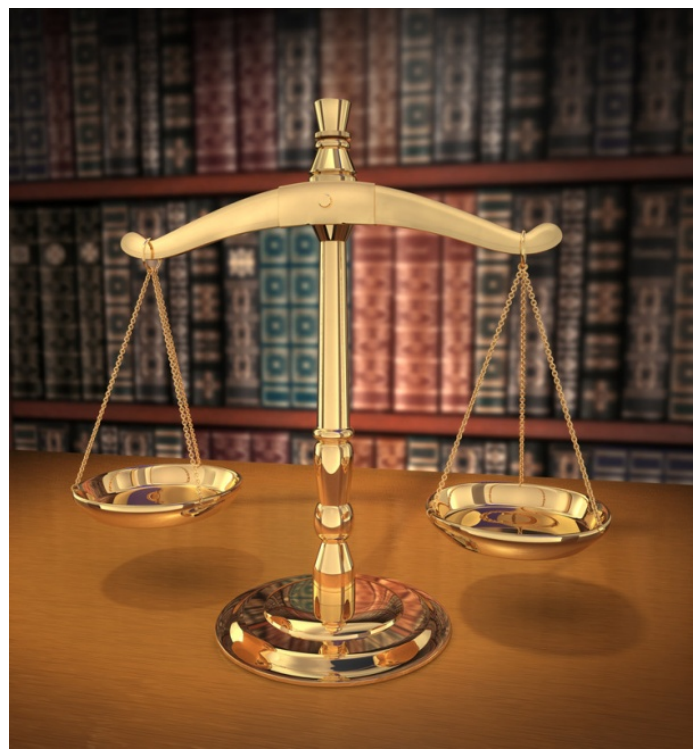

Figure 3: Scales of Justice

unhelpful, and may feel that if they did not consent, their relationship with their physician might be affected, which in turn might impact negatively on the care of their infant. Thirdly, parents' decisions may be affected by a researcher's vested interest in the project. For example, a researcher could have a financial or professional interest in the research and this may influence how information is conveyed. He or she may paint the research in a particularly positive light and stress the possible benefits, while downplaying the potential risks.

These issues can affect the validity of the parent's informed consent. As they may not understand the research or may not receive objective information, their decision may not be fully informed and based on the reasoned balancing of options. In addition, feelings of obligation towards healthcare professionals can mean that their consent is not completely voluntary as parents feel under pressure to make decisions which they would not otherwise make.

\section{Research Aims: What can the Law do?}

Ethical research with neonates must be encouraged, in order to improve their well-being and protect their right to life and health. Law plays an important role in this. A robust regulatory framework can lead to the promotion of medical research, as researchers are protected by defined rules in relation to what constitutes ethical research. Regulation can also contribute to the protection of the rights of parents and their children to autonomy and dignity as researchers must conform to certain ethical standards. However, at present, there is a lack of legal regulation of neonatal medical research in Ireland. Outside the area of clinical trials, there is no legislation governing medical research with neonates. Neither 
are there professional guidelines in relation to obtaining parental consent for a neonate's involvement in medical research.

My thesis will make recommendations for legal reform in the area of neonatal medical research, focusing on the areas of informed consent and the role of Research Ethics Committees. It is important that the issue of informed consent is regulated by legislation, professional guidelines and professional training programs. Consent must be free from the influences of researcher bias and therapeutic misconception, and parents must be given time and support, so that they can adequately protect the rights of their child in the context of neonatal medical research. It is also important that the consent process is informed by children's rights as set out in the Convention on the Rights of the Child 1989, including the child's right to life, the right to health, the right to have their best interests to be considered as a primary consideration, and the right to be free from exploitation. Ireland ratified this Convention in 1992, and thus is bound to protect these rights through legislative and administrative measures under Article 4. While neonatal medical research is focused on parental consent, it must be borne in mind that the informed consent process is primarily about promoting the neonates' rights and ensuring that their interests are protected. The aim of reform should be to ensure that consent procedures respect the inherent dignity of the neonate as a human being. This is done by ensuring that parents are facilitated to make decisions which are in their child's best interests and which protect their human rights.

In summary, it is important that medical research is carried out with neonates in order to promote the right to life and health of this group of children. In order to encourage medical research in this area, Ireland needs to develop law and policy for neonatal medical research. However, there needs to be a balance between promoting research and protecting neonatal human rights. Medical research must be encouraged, but not at the price of the violation of the human rights of a vulnerable population. As Freund notes, it is important to remember that a slower progress in the conquest of diseases may be preferable than having research practices which lead to the erosion of moral values, the loss of which, would make the most dazzling triumphs of scientific progress not worth having (P. Freund, Experimentation with Human Subjects, 1969).

I wish to acknowledge help and support of all the staff of the Faculty of Law and especially my supervisors, Professor Ursula Kilkelly and Dr. Deirdre Madden. I would like also to thank the Law Faculty and the Department of Children and Youth Affairs for providing funding for my PhD. 